\title{
RETOS DE LA EDUCACIÓN SUPERIOR EN AMÉRICA LATINA: EL CASO DE REPÚBLICA DOMINICANA
}

\section{Challenges of Higher Education in Latin America: The Case of Dominican Republic}

\author{
Enrique Sánchez Costa ${ }^{1}$
}

Recibido: 7-6-2016 • Aprobado: 25-1-2017

\begin{abstract}
Resumen
La educación superior en América Latina se encuentra hoy ante desafíos de enorme envergadura. El crecimiento cuantitativo de las universidades latinoamericanas, en términos de alumnado y creación de centros, debería ir acompañado de un incremento parejo de la calidad académica. Este artículo analiza el caso particular de la educación superior en la República Dominicana para, a partir de él, realizar diagnósticos y sugerencias aplicables para el conjunto de América Latina.
\end{abstract}

Algunos de los retos analizados son los desafíos que plantea la globalización y la internacionalización; la tarea urgente de incrementar la cantidad y calidad de la investigación, así como de conectarla con la docencia universitaria; la necesidad de asegurar la formación disciplinar y metodológica de los docentes (en especial, a través del doctorado); así como la importancia que reviste el portal web de las universidades, como forma de interactuar creativamente con los agentes nacionales e internacionales.

Palabras clave: educación superior; universidad; América Latina; República Dominicana.

1. Coordinador del Doctorado en Estudios del Español: Lingüística y Literatura de la Pontificia Universidad Católica Madre y Maestra (PUCMM, Santo Domingo). Correo electrónico: enriquesancos@gmail.com

\begin{abstract}
Higher education in Latin America now faces inmense challenges. The quantitative growth of Latin American universities, in terms of students and center creation, should be accompanied by a steady increase in academic quality. This article analyzes the case of higher education in the Dominican Republic, as an example which allows us to perform diagnostics and suggestions applicable for the whole of Latin America.

Some of the topics discussed here are the challenges of globalization and internationalization; the urgent task of increasing the quantity and quality of research, and connecting it with university teaching; the need to ensure discipline and methodological training of teachers (especially through doctorate); as well as the importance of the university web portal as a way to interact creatively with national and international actors.
\end{abstract}

Keywords: Higher education; university; Latin America; Dominican Republic. 


\section{Introducción}

La educación superior en América Latina creció exponencialmente durante la segunda mitad del siglo xx. Entre el año 1950 y el 2000 el número de instituciones universitarias pasó de 75 a 1.500 , el número de estudiantes de 276.000 a casi 12 millones y la matrícula estudiantil se multiplicó por 45. Con todo, la tasa bruta de escolarización en la educación superior, al filo del año 2000, seguía siendo más baja en América Latina (19\%) que en el promedio de los países desarrollados (51,6\%) (Fernández, 2004, citado en Gazzola \& Didriksson, 2008, p. 28).

La tendencia creciente se intensificó durante la primera década del siglo xxI, gracias, en parte, al incremento de gasto público en educación superior por parte de los gobiernos latinoamericanos (del 4,6\% del PIB en el año 2000 al 5,2\% en 2013) (UNESCO, 2015, p. 19). En 2008, la matrícula estudiantil universitaria se acercaba a los 22 millones y se contabilizaban cerca de 4.000 instituciones universitarias, dos terceras partes de las cuales eran privadas (Brunner \& Ferrada, 2011, p. 113). Cabe destacar, entre otros progresos, la plena incorporación de la mujer al ámbito universitario (cuya matrícula supera hoy, en muchos países de América Latina, a la de varones), así como el creciente acceso de los sectores más humildes a la educación superior (aunque las desigualdades son todavía muy pronunciadas).

Con todo, la expansión cuantitativa de la educación superior en América Latina (hasta el punto, en muchos casos, de la masificación estudiantil) no ha estado acompañada de un crecimiento cualitativo parejo. Llama la atención, en este sentido, la escasa presencia de universidades latinoamericanas en los rankings internacionales más exigentes (que jerarquizan universidades del mundo según estándares de calidad académica claros y contrastados). Así, el Academic Ranking of World Universities o Shanghai Ranking (ARWU, 2015), en su edición de 2015, solo incluye 9 universidades de América Latina entre las 500 mejores del mundo (el 1,8\%).

Ampliando un poco el espectro, el Best Global Universities Rankings (U.S. News and World Report, 2016), en su edición de 2016, enlista 26 universidades latinoamericanas entre las 750 mejores (el 3,4\%). Por su parte, The Times Higher Education World University Rankings (2016) arroja resultados casi idénticos, pues en la edición 2015-2016 posiciona 28 universidades latinoamericanas entre las 800 mejores (el 3,5\%).

Tal como hemos visto, el porcentaje de universidades latinoamericanas emplazadas entre las 800 mejores del mundo es exiguo. Entre las pocas seleccionadas predominan las brasileñas $y$, a cierta distancia, las chilenas, mexicanas y argentinas. En la edición 2015-2016 de un ranking de alcance todavía mayor, el University Ranking by Academic Performance (URAP, 2016), que enlista las 2.000 mejores universidades, se incluyen 100 latinoamericanas (el 5\%). Algunos países caribeños están representados, como Cuba o Puerto Rico; pero, de nuevo, igual que en los otros rankings examinados, las universidades dominicanas quedan fuera de la lista.

Es necesario acudir a un ranking específico de las universidades de América Latina, el QS University Rankings: Latin America (Quacquarelli Symonds, 2016a), para encontrar instituciones dominicanas. En su edición de 2016, este ranking incluye 11 universidades de la República Dominicana. La primera, en el rango 151-160, es la Universidad Iberoamericana (UNIBE); la segunda, en el rango 181-190, es la Pontificia Universidad Católica Madre y Maestra (PUCMM); la tercera y la cuarta, en el rango 251-300, son la Universidad Autónoma de Santo Domingo (UASD) y el Instituto Tecnológico de Santo Domingo (INTEC). Las siete restantes se ubican en el rango 301+.

Es cierto que la educación superior en este país caribeño presenta un nivel de desarrollo inferior al de 
otros países latinoamericanos (como Brasil, México o Chile). Pero, también es verdad que la mayoría de las dificultades y los retos que se presentan en la educación superior dominicana son compartidos -en mayor o menor grado- por los demás países de América Latina. Así, el análisis del caso dominicano servirá como ejemplo concreto a partir del cual realizar propuestas sobre la educación superior que puedan aplicarse -con ligeras variantes- a todos los países de América Latina.

\section{Globalización e internacionalización}

Las primeras universidades medievales (Bolonia, París, Oxford, etc.), constituidas desde finales del siglo XI, nacieron bajo el signo de la universalidad y la unidad de los saberes. No solo se utilizaba una misma lingua franca -el latín- para la comunicación científica (Spinoza o Newton todavía escribirán en latín sus obras, en el siglo XVII), sino que, además, la Universidad medieval posibilitaba la movilidad estudiantil y profesoral. Santo Tomás de Aquino, por ejemplo, que no tuvo reparos en integrar aportaciones de diferentes épocas y tradiciones (Aristóteles, san Agustín, Averroes, Avicena, etc.), estudió en París y Colonia e impartió más tarde docencia en París, Nápoles, Orvieto, Roma y Viterbo.

Durante el siglo XIX, al consolidarse en Europa los Estados Nacionales, la Universidad quedó absorbida por el Estado-Nación. Emergieron las grandes universidades investigadoras estatales (comenzando, en 1810, por la entonces llamada Universidad de Berlín). Aunque el modelo alemán se extendió por toda Europa, cada Estado trató de proteger su sistema universitario de influencias foráneas. El localismo triunfaba sobre la universalidad. Y, si durante los dos primeros tercios del siglo XX continuó la movilidad estudiantil y profesoral, se debió sobre todo a exilios voluntarios o forzosos, fruto de seísmos políticos y militares (como el exilio de intelectuales españoles republicanos en América Latina o el de intelectuales judíos europeos en Estados Unidos). La movilidad seguía existiendo, pero no era una tendencia general.

Desde las últimas décadas del siglo xx, y con mayor fuerza desde la caída del muro de Berlín, se desarrolló en el mundo el proceso de globalización; es decir, la creciente comunicación e interdependencia entre las sociedades, las culturas y los mercados de los distintos países del mundo. Junto a los cambios políticos (descolonización, apertura económica de China, caída del bloque soviético, mayor democratización de América Latina, creación y consolidación de la Unión Europea, etc.), la globalización ha estado propiciada por las revoluciones tecnológicas de las últimas décadas. Entre ellas, la transformación de las tecnologías de transporte, las comunicaciones y la informática, especialmente internet que, aunque nace como tecnología en los años sesenta, se abre al público a partir de 1993 y comienza a extenderse masivamente al filo del año 2000 .

Si la globalización hace referencia a las tendencias globales de que venimos hablando, la internacionalización, tal como la define un informe de la UNESCO de 2009, es "la diversidad de políticas y programas que las Universidades y los gobiernos implementan para responder al fenómeno de la globalización" (Altbach, Reisberg \& Rumbley, 2009, p. 7). Una de las mayores manifestaciones de la internacionalización en la educación superior es el incremento notable de la movilidad estudiantil. En el año 2000 estudiaron fuera de su país de origen 1,8 millones de personas. En 2007 la cifra era de 2,8 millones (un incremento del 53\% respecto al dato anterior) y en 2013 la cifra alcanzaba los 4,1 millones de estudiantes (el 1,8\% de la matrícula estudiantil) (p. 25). 
Expongamos ahora las cifras más relevantes de movilidad estudiantil internacional en la educación superior, según datos de 2013 que presenta el UNESCO Institute for Statistics (2013). En ese año, los ratios de estudiantes que realizaron estudios en el extranjero fueron, según regiones de procedencia: Asia Central (7,6\%), África Subsahariana $(4,0 \%)$, Asia Occidental y del Sur (1,0\%), América Latina y el Caribe $(0,9 \%)$ y Norteamérica $(0,5 \%)$. Desde la perspectiva inversa, las regiones que acogieron un mayor número de estudiantes extranjeros fueron: Norteamérica y Europa Occidental (57\% del total de estudiantes en movilidad internacional), Asia Oriental y el Pacífico (19\%) y Europa Central y del Este (10\%). Llama la atención, en este sentido, la baja movilidad estudiantil en América Latina, por debajo de otras regiones menos desarrolladas.

República Dominicana, por su parte, envió en el año 2013 a 4.360 estudiantes al extranjero, lo que representa una tasa de movilidad estudiantil en el país del 0,8\% (muy similar a la tasa regional de América Latina). Los seis países que recibieron más de cien estudiantes dominicanos fueron: España (1.747), Estados Unidos (1.660), Cuba (254), Francia (137), Italia (121) y Canadá (114). No extraña la primacía de España y Estados Unidos como países receptores de estudiantes dominicanos, por el prestigio de ambos países y, en el caso de España, por la lengua común. Por otro lado, República Dominicana acogió ese año a 10.676 estudiantes internacionales, lo que representa una tasa de estudiantes internacionales del $2,3 \%$.

En contraste, los seis países que enviaron más de cien estudiantes a República Dominicana fueron: Haití (6.945), Estados Unidos (1.962), Venezuela (185), Colombia (185), Cuba (169) y España (101). Es natural que Haití, por su condición limítrofe con República Dominicana, así como por su inferior desarrollo económico y académico, sea el principal exportador de estudiantes universitarios.
Es relevante, también, el número de alumnos estadounidenses recibidos (muchos de ellos, en programas de inmersión lingüística), así como el potencial de crecimiento y de oportunidades académicas que implica.

Junto a la movilidad estudiantil (que, añadimos, también es muy elevada dentro de Europa, gracias al programa ERASMUS de la Unión Europea), la internacionalización ha incrementado igualmente la movilidad del otro gran agente de la educación: los docentes. El mercado laboral académico se está globalizando. Miles de profesores están cruzando fronteras para asumir posiciones académicas a todos los niveles. Muchos de ellos lo hacen a través de becas (gubernamentales o no), a menudo para investigar en países con laboratorios más avanzados y bibliotecas mejor surtidas. Otros lo hacen buscando mejores salarios y condiciones laborales, viajando, habitualmente, del Sur al Norte. Además, la globalización permite que, de alguna manera, se autorregule el mercado laboral docente: mientras que en Europa hay pocas plazas docentes y un exceso de profesores cualificados, América Latina los necesita con urgencia.

Otro gran ámbito en el que la globalización ha transformado la política universitaria es en la creciente proliferación de convenios y acuerdos entre instituciones de diversos países. Este fenómeno ha impactado tanto en el nivel de la gestión gubernamental como en el de la gestión de cada universidad. En el ámbito público, el mayor proceso de integración académica ha sido desarrollado por la Unión Europea. Esta, a partir de la Declaración de Bolonia (1999), ha llegado a integrar a más de 40 países en el Espacio Europeo de Educación Superior (EEES) y a homologar los currículos, los títulos y las calificaciones -de grado y postgrado- de todas las universidades del EEES.

En este sentido, desde hace unos años se está tratando de crear, por medio del IESALC, el "Espacio 
de la Educación Superior en América Latina y el Caribe", que se articuló a través de ENLACES. Todavía no se han logrado avances significativos en este proyecto, tan ambicioso como difícilmente realizable, pues, a diferencia de lo que sucede en Europa, los países latinoamericanos no comparten una integración política pareja a la Unión Europea.

Todas las universidades importantes del mundo han establecido numerosos vínculos con otras instituciones universitarias y otros centros de investigación. En el mundo interconectado en el que vivimos, es necesario crear sinergias y acuerdos de beneficio mutuo con instituciones de otros países. Los programas de doble titulación (en grado y, sobre todo, en postgrado), son hoy una realidad en cualquier universidad valiosa. Los hermanamientos $y$ acuerdos entre universidades afectan a los programas, pero también al intercambio y a la circulación de docentes, que resulta muy beneficiosa para la Universidad.

La internacionalización debe ser, hoy en día, uno de los ejes principales de cualquier universidad. En nuestro mundo globalizado no puede concebirse la Universidad solo en un contexto local y nacional: es preciso pensarla también en un ámbito o campo de juego internacional. Cada institución universitaria no compite únicamente con las demás universidades de su población o país, sino con las de otras regiones del mundo.

Lo antes expuesto revela una realidad que refleja la importancia concedida a los rankings (pues encauzan las decisiones de ingreso de estudiantes tanto nacionales como internacionales), así como la creciente población de estudiantes internacionales. Existe, cada vez más, un mercado global universitario del que las universidades latinoamericanas no pueden sustraerse si no quieren ser arrumbadas hacia la periferia y la irrelevancia.

\section{Investigación}

Las tres funciones esenciales de las instituciones de educación superior son la investigación, la docencia y la extensión universitaria, es decir, la vinculación de la Universidad con la sociedad. Y las tres deberían desarrollarse en todas las universidades, pues se necesitan y se refuerzan mutuamente. $\mathrm{Y}$ es que, como aclara Alejandro Guzmán: "La Universidad enseña y difunde lo que investiga; investiga para enseñar y difundir, y difunde y enseña porque investiga. Entonces, desde el momento en que dentro de la Universidad la investigación es fuente de la docencia y de la difusión, bien puede hablarse de Universidad investigadora" (Rojas, 2012, p. 82).

No hay auténtica docencia universitaria sin investigación. Pues, al cabo, nadie da lo que no tiene, ni enseña lo que no sabe. El conocimiento teórico no se adquiere por ciencia infusa. Nuestra mente necesita del esfuerzo intelectual, constante y arduo, para asimilar los conocimientos del pasado y del presente, y para pergeñar la ciencia del futuro. La Universidad, precisamente, se dedica a la creación, adquisición y transmisión de nuevo conocimiento (riguroso y científico). Son todas ellas operaciones ligadas entre sí, que, como los filamentos metálicos de una gran cadena, se entreveran y potencian mutuamente.

En un sentido parecido, definía el filósofo Julián Marías la Universidad como "la institución cuya finalidad primaria es enseñar a pensar. [...] La única justificación de los profesores es que pueden contagiar el pensamiento a los estudiantes, y esto sólo puede hacerse pensando ante ellos, con ellos" (Rojas, 2012, p. 34).

La excelencia docente no es posible si, previamente, el profesor no la ha experimentado y encarnado en su actividad investigadora. Él es el capitán que debe guiar el barco (con la participación de toda la tripulación, de todos los estudiantes) para alcanzar la terra incognita del conocimiento. Lo expresa, 
con otras palabras, Alejandro Llano (2012): la Universidad es "una empresa de indagación compartida, cuya finalidad es encontrar lo bueno y lo mejor a través del avance en el conocimiento". Una indagación que comparte dinámicamente el profesor con sus alumnos -buscando la retroalimentación-, así como con sus colegas académicos de su propia universidad, de otras universidades del país y del resto del mundo.

Hoy en día, se tiende a clasificar a las universidades según el tipo de docencia que imparten y, más todavía, según el nivel de su compromiso con la investigación. En la base de la jerarquía de instituciones de educación superior encontraríamos los centros de formación vocacional, que ofrecen programas de dos o tres años, con un claro fin profesionalizador. Más arriba se situarían las universidades orientadas exclusivamente a la enseñanza (sobre todo a alumnos de grado).

$\mathrm{Al}$ no tener que dedicar recursos a la investigación y al pagar poco a sus docentes -muy sobrecargados de horas de clase- estas universidades pueden ofertar titulaciones académicas a un costo más reducido que otras. Por esta razón y por la masificación universitaria que se está produciendo en los países en vías de desarrollo (muchos jóvenes desean acceder a la Universidad, aunque pocos cuentan con recursos financieros) estas universidades dedicadas exclusivamente a la docencia son las que están experimentando un mayor crecimiento en América Latina.

Todavía más arriba en la jerarquía encontramos las universidades orientadas tanto a la enseñanza como a la investigación. En general, en dichas instituciones suele primar la docencia, aunque también reservan un cierto papel para la investigación. Pienso que instituciones dominicanas como UNIBE, la PUCMM o el INTEC podrían encuadrarse en este tipo de universidades que forman tanto a alumnos de grado como de postgrado (especialidad, maestría y, en algunas pocas disciplinas, también doctorado).
En la cima de la jerarquía de instituciones de educación superior se sitúan las universidades investigadoras, caracterizadas por su énfasis en la investigación (a la que dedican cuantiosos recursos administrativos y financieros). Por eso es en dichas universidades donde tienden a formarse las nuevas generaciones de investigadores, a través de maestrías orientadas a la investigación y, sobre todo, de programas de doctorado.

En América Latina, como destaca el Informe de la UNESCO de 2009, la investigación se suele llevar a cabo en unas pocas universidades -casi siempre públicas y de enorme tamaño-, como la Universidade de São Paulo en Brasil, la Universidad Nacional Autónoma de México, la Universidad de Chile, la Universidad Nacional de Colombia o la Universidad de Buenos Aires.

Como consecuencia de lo antes expuesto, tan pocas universidades latinoamericanas se sitúan en los puestos altos de los rankings internacionales que miden criterios como el número de papers publicados por profesor, las citaciones que reciben esos $p a-$ pers, el prestigio internacional de las universidades o los contactos con profesores y centros de investigación internacionales. Además, dentro de estas pocas universidades investigadoras latinoamericanas, la investigación se realiza a menudo en centros específicos y alejados de la actividad docente del primer ciclo universitario. Veamos algunos ejemplos de este fenómeno de concentración:

Una universidad pública es responsable del $37 \%$ de todos los papers en Chile; de modo parecido, una universidad produce el 30\% de los papers en México; una sola universidad produce el 25\% de los papers en Brasil, y una sola institución es responsable del 18\% en Argentina. En Chile, por ejemplo, donde las universidades son el actor principal en la producción de ciencia, las cinco instituciones más antiguas llevan a cabo el $80 \%$ de la investigación desarrollada en el 
país (Altbach, Reisberg \& Rumbley, 2009, p. 143).

¿Y cuál es el estado de la investigación en las instituciones de educación superior dominicanas? El Plan Decenal de Educación Superior 2008-2018, elaborado por la Secretaría de Estado de Educación Superior, Ciencia y Tecnología, es claro al respecto. Según este documento, el espacio que ocupa la investigación en la Universidad dominicana "es casi asistemático y sin la decisión y suficiencia que de ella requieren el nuevo orden nacional e internacional de la economía, del mercado, de la competitividad y del desarrollo económico" (SEESCyT, 2008, p. 83).

El documento, tras recalcar "que la mayoría de universidades están ancladas" en "la sola función de docencia", advierte: "Si en la actualidad, y hacia futuro, la Universidad no investiga y produce conocimiento mediante la investigación, su accionar es estéril y finalmente su existencia no tiene sentido; además, éticamente engaña a sus estudiantes y al país" (p. 84).

Entre las causas de la escasa producción investigadora de los profesores en República Dominicana (reflejada en el débil posicionamiento de sus universidades en los rankings internacionales) habría que destacar, en primer lugar, que la mayor parte de los profesores no han sido formados en una auténtica cultura académica de la investigación. Muchos de ellos desconocen que la investigación es una de las funciones esenciales de la Universidad y que es irrenunciable para todo profesor universitario que se precie. Y, entre los profesores que sí han oído el término investigación, muchos no saben en realidad qué significa; desconocen, por ejemplo, las particularidades de los principales géneros textuales científico-académicos, así como su producción y difusión a través de publicaciones en soporte físico o digital.
Incluso los profesores universitarios que son conscientes de la trascendencia de la investigación académica experimentan serias dificultades para llevarla a cabo. La principal, seguramente, es la sobrecarga de docencia. Un profesor que imparte 20, 30 o 40 horas semanales de docencia en la Universidad (como es habitual en República Dominicana) es difícil que disponga de tiempo de calidad para leer críticamente publicaciones científicas de gran densidad conceptual.

Ampliando lo anterior, en muchos casos, los profesores por asignatura o de medio tiempo -que son la inmensa mayoría - trabajan por necesidad económica en varias universidades (el llamado, coloquialmente, "profesor-taxi"), lo que contribuye a un mayor gasto de energías físicas y a una mayor dispersión intelectual. Así, a muchos profesores les faltan horas disponibles y fuerzas mentales para dedicarlas al trabajo arduo de la investigación.

Si hablamos del impacto de la sobrecarga docente en la escasa investigación de los profesores, conviene considerar también cómo influyen las teorías pedagógicas actuales en la productividad investigadora. Frente a la enseñanza magistral tradicional, jerárquica y memorística, se insiste hoy en el aprendizaje comunitario, práctico y continuo. Esta educación continua y personalizada (numerosas evaluaciones por período, valoración de muchas competencias, etc.) supone para el profesor incesantes correcciones de trabajos de clase, pruebas y evaluaciones.

Lo que se busca es, de hecho, aplicar a la Universidad la educación continua que se ha implementado tradicionalmente en la enseñanza primaria y secundaria. Ello conlleva efectos beneficiosos para los alumnos, pero también un problema: los profesores de colegio, salvo excepciones, no investigan. ¿Cómo iban a hacerlo, si tienen que impartir tantas horas diarias de clase y, a continuación, llevarse trabajo a casa para corregir? De ahí que la aplicación de la educación 
continua en la Universidad, si se lleva a un grado extremo, puede levantar un serio obstáculo para el desarrollo de la investigación.

En las instituciones superiores de República Dominicana (y en gran parte de las de América Latina) no suele encontrarse una cultura de la investigación potente. Los motivos son muy variados y tienen casi siempre fuertes raíces en los problemas económicos, políticos y culturales que han afectado a los países latinoamericanos durante las últimas décadas. Del mismo modo que, como es sabido, cuando hay pocos recursos educativos los gobiernos suelen sacrificar la educación superior en beneficio de la educación inicial (y, en menor grado, de la educación media), cuando hay pocos recursos en la universidad se suele sacrificar el postgrado en beneficio del grado, o la investigación en beneficio de la docencia. El planteamiento tiene su lógica (se busca asegurar la base o el soporte de la estructura educativa), pero también tiene sus peligros.

Es imperativo implantar una sólida cultura de la investigación que, con el paso del tiempo, acabe afectando a la docencia en todos los niveles educativos. Al cabo, la calidad académica de la educación inicial y media depende del nivel académico de sus docentes, quienes se forman en la universidad. Y estos solo recibirán una formación adecuada si sus profesores universitarios son, ellos mismos, buenos investigadores. Algo difícil de conseguir y que requiere que estos, a su vez, hayan sido formados -a través del doctorado- por excelentes profesores investigadores. A pesar de las dificultades, si hay esfuerzo por parte de todos los agentes (Gobierno, universidad, sociedad civil e industria), la luz del conocimiento siempre acaba por alcanzar a todos; a los que están más cerca y, si esta es potente, también a los que están más lejos. Pero, para poder ver, hay que prender la luz de la investigación.

\section{Docencia}

El capital principal de una universidad es el cuerpo de profesores de la institución. Y su excelencia y prestigio dependerán, en última instancia, de la capacidad que tenga por atraer y retener a los mejores docentes en cada área del saber. Algo que se propicia, por supuesto, con buenos salarios; pero también con otros muchos factores que van más allá del factor económico: la autoridad, el respaldo y la confianza que se otorga a los profesores en la universidad, el respeto a la libertad de cátedra, las oportunidades que se les ofrecen de labrarse una carrera académica exitosa, de trabajar en un ambiente ilusionante en los aspectos materiales y humanos, etc.

Todo gran profesor universitario es un apasionado de su disciplina y se sirve de su pasión intelectual para lograr la difícil tarea de estar siempre actualizado en su área de conocimiento. Es una persona que, como escribió Étienne Gilson, conoce "prácticamente todo lo que, al presente, puede conocerse en su campo, que se da cuenta de su propia ignorancia y que trabaja muy duro para disminuirla". Aclara Gilson que no se refiere tanto al volumen de conocimientos, sino a la actitud propia de quien "ha decidido, de una vez por todas, aplicar las exigencias de su conciencia moral a su vida intelectual" (Rojas, 2012, p. 21). La humildad intelectual -el reconocimiento socrático de la propia ignorancia-, unida a la pasión por la verdad, por el conocimiento disciplinar e interdisciplinar, son los dos grandes motores que impulsan el desarrollo académico de una persona.

La solvencia intelectual, académica y profesional de un docente le concederá una autoridad natural ante sus alumnos. El propio esfuerzo del profesor por mantenerse actualizado en su disciplina le proporcionará, además, virtudes pedagógicas: la experiencia propia de lo difícil que puede resultar, en ocasiones, la comprensión de ciertos temas; el conocimiento de los callejones sin salida en el mapa 
del saber humano, así como de los mejores caminos y atajos para alcanzar los objetivos de aprendizaje. No será un capitán retirado que aconseja desde la lejanía a sus pupilos, sino un capitán activo, embarcado con sus alumnos en la aventura del conocimiento: en la comprensión de las verdades teóricas y prácticas de una disciplina.

Junto al dominio portentoso de su disciplina y la facilidad para desbrozar ante los alumnos los caminos más intrincados del saber, los mejores docentes universitarios buscan que sus estudiantes aprendan de forma duradera y profunda. Que las realidades aprendidas no reposen simplemente en la memoria, sino que conmuevan la inteligencia, el corazón y el alma de los alumnos; que cambien su manera de pensar y de actuar; que crezcan y se desarrollen como semillas en tierra fértil. Para lograrlo, los mejores profesores no solo ofrecen a sus alumnos la ciencia más actualizada, sino que la confrontan con las realidades acuciantes del mundo profesional, con las enseñanzas que les ofrece su experiencia práctica, así como con los debates más relevantes que acontecen hoy en la Academia (Bain, 2005, pp. 26-28).

Además, el profesor de universidad, puesto que trabaja con la realidad más sagrada y quebradiza, con la persona humana, debe ser un forjador de libertad y un sembrador de esperanza. De ahí que, George Steiner (2007, p. 135) haya afirmado que "un maestro es el celoso amante de lo que podría ser", pues en cada alumno no ve solo el boceto que ya es sino, sobre todo, la obra maestra que podría llegar a ser. El docente que vive su vocación profesional dedicará todos sus esfuerzos a fomentar esa obra maestra en los alumnos, proponiendo a sus estudiantes metas y horizontes audaces, para que lleguen donde, quizá, no osarían llegar. Ese docente sobresaliente, del mismo modo que ha avanzado en su vida profesional exigiéndose al máximo, espolea a sus alumnos para que expandan sus potencialidades.
Uno de los problemas que experimenta la docencia universitaria en América Latina (igual que sucede, de modo creciente, en Norteamérica y Europa) es la precariedad laboral de los profesores. El 80\% de los profesores de educación superior en América Latina (el 82,5\% en República Dominicana, según datos del año 2011) (MESCyT, 2011, p. 208) tienen contrato a tiempo parcial. Las universidades, por motivos económicos, rehúyen los contratos de tiempo completo (que implican un puesto laboral y una remuneración estables, un despacho de trabajo, unos beneficios sanitarios y educativos, etc.).

Estas universidades prefieren -utilizando un término empresarial- externalizar su docencia, recurriendo a profesores sin contrato fijo y, por tanto, prescindibles en términos académicos y laborales. No puede extrañar, en consecuencia, que esos profesores no se sientan identificados con la institución y, a menudo, no estén motivados en su docencia. Al fin y al cabo, ¿qué vocación profesional auténtica se desarrolla a tiempo parcial y de modo intermitente?

Para aumentar la calidad de la docencia es necesario que aumente la calidad de los contratos laborales docentes. Tras un tiempo prudencial de prueba, se debería ofrecer a los profesores un contrato de tiempo completo o, al menos, de medio tiempo. Y, una vez la universidad hubiera ofrecido esos beneficios contractuales al profesor, estaría en condiciones de pedirle reciprocidad en su entrega; de exigirle la máxima implicación en la vida departamental, en las actividades docentes, en la atención a los alumnos, en su formación continua y, ante todo, en su investigación y en su docencia. Solo por medio de profesores apasionados con la docencia e identificados con su universidad puede una institución tejer una identidad corporativa reconocible desde el punto de vista académico y humano.

Otro campo en el que se puede avanzar mucho es en la internacionalización de los docentes. Como apuntábamos en el primer apartado, la universidad debe 
responder a los retos del mundo global por medio de la internacionalización. En este caso, a través de la contratación -temporal o permanente- de profesores extranjeros. Contar con un número adecuado de "profesores visitantes" e incorporar docentes extranjeros contribuye a dinamizar la vida universitaria, tanto en el aspecto investigador como docente.

Toda institución universitaria está siempre tentada por los agujeros negros del localismo, el amiguismo, el nepotismo y la endogamia. Esos vicios académicos, que esclerotizan la vida universitaria e impiden toda renovación, pueden ser combatidos, entre otros caminos, por la convocatoria libre de plazas docentes (debidamente publicitadas en los portales web académicos), que favorecen la movilidad profesoral.

Actualmente, numerosas universidades del mundo han concedido a sus departamentos administrativos el poder sobre las decisiones estratégicas de la institución. La burocratización de la universidad, el triunfo de la letra sobre el espíritu, del proceso sobre la finalidad, está carcomiendo la vitalidad de muchas instituciones de educación superior. Se persigue la reducción de costos por cualquier medio, el incremento de la matrícula universitaria (sin importar el perfil de ingreso de los nuevos alumnos), la maximización del beneficio económico, etc.

Esta visión utilitarista, pragmática y a menudo cortoplacista, es incompatible -si se aplica con radicalidad- con el florecimiento de la vida académica. Es lógico que las instituciones universitarias privadas busquen un beneficio económico y que las universidades públicas traten de ajustar sus presupuestos, pero no al precio de desatender las necesidades de los docentes y el fin de la universidad. El corazón de la vida universitaria no son sus administradores o burócratas, sino sus profesores y alumnos.

Puesto que los docentes son la piedra angular de la institución académica, las universidades deberían realizar la mayor inversión posible para buscar y contratar a los mejores; a profesores con aptitudes pedagógicas y con una enorme pericia disciplinar, que debe resultar patente en su formación académica y en sus publicaciones. Ya hemos hablado, en el anterior apartado, de la importancia de la investigación para la vida universitaria en general y para la docencia en particular. Incidamos ahora en la formación académica y, de modo especial, en el doctorado.

El doctorado es "el más prestigioso e internacional de los títulos académicos" (Nerad \& Evans, 2014, p.5), que acredita la solvencia metodológica, científica y disciplinar de un académico. Es el estudio de postgrado de máximo nivel, forma investigadores capaces de aprehender la ciencia existente y transformarla creativamente, para generar aportaciones originales en una disciplina.

Por lo general, en Estados Unidos y en Europa el doctorado marca el inicio de la carrera académica. Estar cursando un doctorado es allí un requisito casi indispensable para comenzar a impartir docencia y sin la posesión del título de doctor no se puede obtener ninguna plaza académica docente de nivel medio o alto. En América Latina, debido a sus particularidades económicas y socioculturales, la situación es bastante diferente.

En este sentido, el caso de República Dominicana es llamativo, pero no muy distinto al de otros países de la región. Según los últimos datos publicados, en el año 2010 solo el 3,05\% de los docentes de educación superior del país poseían el título de doctor (MESCyT, 2011, p. 202). De hecho, ese mismo año no había en el país ningún alumno matriculado en un programa de doctorado ( $\mathrm{p}$. 119). Hubo que esperar hasta el curso 2015-2016 para que una universidad dominicana, la Pontificia Universidad Católica Madre y Maestra, ofreciera el primer doctorado propio del país: el programa de Doctorado en Estudios del Español: Lingüística y Literatura.

América Latina necesita contar con muchos más doctores entre sus docentes universitarios. Sin 
doctores, la investigación se resiente, pues es el doctorado la principal escuela de formación de investigadores competentes en todos los campos del saber. Por eso el doctorado es tan largo y demandante: porque exige del doctorando una aportación investigadora sólida, personal y creativa en la disciplina en cuestión. Es preciso formar buenos doctores con urgencia, tanto en el extranjero como en los países de origen, y esto último requiere ofrecer un mayor número de programas de doctorado de calidad en América Latina.

Es tan importante la cuestión antes planteada que la agencia Quacquarelli Symonds (QS), en su ranking que enlista las 500 mejores universidades de América Latina, introdujo un criterio de medición no utilizado en el ranking general: el porcentaje de profesores con doctorado. Según la agencia, este criterio "fue identificado como una prueba de rendimiento particularmente significativa para las universidades de la región" (Quacquarelli Symonds, 2016b).

\section{El portal web de las universidades}

Podría sorprender, en un primer momento, que dedicáramos uno de los cuatro apartados de este artículo al portal web de las universidades. Con esto, por supuesto, no queremos equiparar esta cuestión a otras más trascendentales (relacionadas, por ejemplo, con la docencia y la investigación), sino que buscamos resaltar la presencia web de las universidades como uno de los retos que deberían enfrentar hoy las instituciones de educación superior en América Latina. Tal como apunta el Laboratorio de Cibermetría del Consejo Superior de Investigaciones Científicas (CSIC, España):

La Web es clave para el futuro de todas las misiones de la universidad, puesto que es de hecho la principal herramienta de comunicación académica, el canal primordial para desarrollar la educación a distancia, un espacio para la participación de la comunidad y el escaparate abierto al mundo para atraer talento, financiación y recursos de todo tipo (CSIC, 2016a).

Hoy en día, el portal web de una universidad es, como su nombre indica, la puerta de entrada a la institución. En nuestro mundo tecnologizado y global, lo que no está en Internet no "existe". O, por lo menos, no posee la misma entidad que si tuviera una presencia web adecuada. Pensemos en un caso concreto: ¿cuántas personas buscan hoy los datos de un comercio o de cualquier institución en una agenda o guía telefónica de papel y cuántos lo hacen a través de Internet? Esta omnipresencia de Internet es más acentuada, todavía, entre la gente joven. Como certifican muchas encuestas y como muestra la experiencia diaria, hoy en día los jóvenes pasan más tiempo delante de la pantalla de la computadora, el smartphone o el tablet que delante de la televisión.

El portal web de la universidad es hoy uno de los ejes de cualquier institución de educación superior. Y no solo porque algunos rankings influyentes, como el del QS en América Latina, concedan un $10 \%$ de valor al impacto del portal web institucional (que consideran "una forma de evaluar el compromiso de las instituciones para relacionarse con una audiencia global y promoverse a sí mismas internacionalmente") (CSIC, 2016a), sino porque ese valor que reflejan los rankings lo concede, en primer lugar, la sociedad. Muchos estudiantes de término escolar consultan los portales Web de las instituciones de educación superior antes de escoger su universidad. La presencia e impacto web de una institución universitaria influye de modo directo en el número de estudiantes que ingresan cada año en ella.

Además, el portal web es la tarjeta de presentación nacional e internacional de una universidad. A la hora de firmar convenios con instituciones académicas, empresas, fundaciones, organismos gubernamentales o no gubernamentales, etc., el portal 
web de la universidad es lo primero que se observa. Y sabemos que la primera impresión -o imagen- que recibimos de algo o de alguien es la más importante. La que quedará en el subconsciente e influirá -a través del prejuicio- en todos nuestros juicios posteriores. De ahí que se haya definido la retórica (o el arte de la persuasión) como el arte o la técnica de crear presencias. Pues bien: hoy en día el portal web es el elemento más importante a la hora de crear presencias positivas que perduren en la consciencia -y subconsciencia- de la persona, en la de posibles alumnos, profesores, empresas, gobiernos y asociaciones que puedan interactuar con la universidad.

El Laboratorio de Cibermetría del CSIC, antes citado, publica desde 2004 -cada enero y julio- el "Ranking Web (Webometrics) de Universidades". Dicho ranking se sirve de las técnicas de una nueva disciplina, la Cibermetría o Webometría, para analizar cuantitativamente los contenidos relacionados con la generación y comunicación académica del conocimiento científico. El ranking se construye a partir de dos indicadores, con un valor del $50 \%$ cada uno.

El primer indicador es la visibilidad web o impacto, que evalúa todos los enlaces entrantes que recibe el dominio web universitario por parte de terceros (académicos o no); muestra el prestigio institucional, el rendimiento académico, el valor de la información y de los servicios del dominio. El segundo indicador, la actividad, valora la presencia (número de páginas web alojadas en el dominio web principal de la universidad, incluyendo los subdominios y directorios), la apertura (número de ficheros ricos -pdf, doc, docx, ppt- publicados en el dominio web) y la excelencia (papers publicados en revistas de alto impacto) de un portal web (CSIC, 2016a).

La última edición del ranking, de enero de 2016, incluye más de 21.000 universidades. Entre las 500 primeras encontramos solo 14 universidades latinoamericanas (el 2,8\%) (CSIC, 2016b): 9 de
Brasil, 2 de Chile y de Argentina y 1 de México. De todas ellas, solo la Universidade de São Paulo (49) y la Universidad Nacional Autónoma de México (62) se encuentran entre las 100 primeras. La tercera universidad latinoamericana mejor posicionada es la Universidade Estadual de Campinas (194), la cuarta es la Universidad de Chile (272) y la quinta es la Universidad de Buenos Aires (295).

Las nueve universidades restantes se encuentran entre los puestos mundiales 300 y 500. También en el espacio digital, igual que en los rankings generales antes reseñados, las universidades latinoamericanas -salvo contadas excepciones- demuestran estar todavía lejos de los estándares más elevados de exigencia académica.

$\mathrm{Si}$ atendemos al posicionamiento de las universidades dominicanas, el panorama es todavía más desalentador. La mejor posicionada, la UASD, aparece en el puesto 4.579 del ranking mundial. Las otras cuatro que conforman las cinco primeras del país son: el INTEC (4.674), la UNPHU (5.856), la PUCMM (6.006) y UNIBE (8.045). Estos posicionamientos no son casuales: la realidad es que en los portales web de las universidades dominicanas es casi imposible encontrar los perfiles web de los profesores $y$, a menudo, ni siquiera los de los directores de departamentos.

Por otro lado, si contrastamos las cinco primeras universidades del ranking QS University Rankings: Latin America con las cinco primeras del ranking mundial, cuatro de ellas coinciden (UASD, INTEC, PUCMM y UNIBE), aunque aparezcan en distinto orden. Se trata de una coincidencia relevante, por cuanto muestra la fiabilidad de los criterios y las mediciones de estos rankings.

El portal web de una universidad es, hoy en día, su tarjeta de presentación nacional e internacional y su mejor plataforma para interactuar con estudiantes, profesores, empresas, gobiernos, fundaciones y demás agentes sociales. La presencia web de una universidad impacta en su conocimiento y prestigio 
nacional e internacional; y, a través de él, en el número de alumnos de nuevo ingreso. También influye en el modo en que se difunde y promociona la investigación en la universidad, tanto a nivel colectivo como individual. Por tanto, es fundamental que una institución universitaria cuente con un portal web de primer nivel, tanto en cantidad (número de páginas y archivos) como en calidad y accesibilidad de esas páginas y esos archivos.

Es imperativo impulsar el desarrollo de los portales web de las universidades latinoamericanas. Debería acrecentarse en gran medida el número de páginas alojadas y mejorar la calidad, variedad y actualización de los contenidos. Una recomendación concreta es que todos los centros de investigación y todos los departamentos académicos de las universidades tengan su propio subportal o subsite dentro del portal web de la universidad.

Asimismo, es recomendable que cada uno de estos subportales departamentales incluya, al menos: una página de bienvenida y de explicación sobre la misión y visión del departamento; una página donde se muestre el equipo docente y administrativo, así como una página personal para cada profesor (que muestre sus datos de contacto profesional, su formación académica, sus asignaturas impartidas, sus líneas de investigación y publicaciones recientes); una página con las noticias del departamento, con enlaces valiosos y datos de contacto.

\section{Conclusión}

Durante las últimas décadas, América Latina ha sido testigo de la incorporación plena de la mujer a la universidad, así como del acceso a la educación superior de capas cada vez más amplias de población. Estas realidades positivas, unidas a la explosión mundial de población, han generado el problema de la masificación en las aulas universitarias. Por un lado, las grandes universidades estatales están hoy abarrotadas; en la Universidad Autónoma de
Santo Domingo, por ejemplo, muchos estudiantes se ven obligados a recibir las clases de pie, por falta de espacio y de pupitres.

Por otro lado, para atender la creciente demanda estudiantil, se han multiplicado las universidades privadas, hasta el punto de que, en 2011, cerca del $52 \%$ de la matrícula de educación superior en América Latina correspondía a las instituciones privadas (SITEAL, 2011, p. 5). Junto a muchas universidades privadas de calidad, también abundan las que son solo expedidoras de títulos de licenciados; universidades dedicadas a la docencia intensiva, movidas por el afán de lucro y ajenas a los criterios más básicos de calidad académica.

Ante la multiplicación del cuerpo de estudiantes, cada vez más heterogéneo (en términos de edad, formación previa, expectativas, etc.), así como ante los desafíos del mundo actual, las universidades latinoamericanas -públicas y privadas- se enfrentan a numerosos retos. En primer lugar, la internacionalización: la adecuación de la universidad a la aldea global, al mundo interconectado e internacionalmente competitivo del siglo XxI. Urge impulsar una mayor movilidad internacional de profesores y estudiantes (todavía reducida en América Latina), así como la creación de redes y grupos de investigación, programas de postgrado compartidos y formas de colaboración científica y tecnológica entre universidades nacionales e internacionales.

La investigación es, tal vez, el mayor reto al que se enfrentan hoy las universidades en América Latina. En muchos casos falta una cultura sólida de la investigación, que la conciba unida inextricablemente a la calidad de la docencia. Es preciso multiplicar las universidades que, además de la docencia, dedican también recursos significativos a la investigación. Deben crearse más y mejores revistas académicas; deben formarse a muchos más profesores en la metodología de la investigación y en la producción de textos científicos. $Y$, ante todo, debe liberarse de horas de docencia a los profesores, para que puedan 
dedicar energías y tiempo de calidad a la investigación. Este debería ser un requisito para acceder, en la universidad, a cualquier posición docente.

El capital principal de una institución universitaria es su cuerpo de profesores; su solvencia intelectual y académica, su pericia investigadora y su capacidad docente. En el campo de la docencia universitaria también existen, en América Latina, numerosos retos que acometer. En primer lugar, es preciso reducir la precariedad laboral de los docentes universitarios (la inmensa mayoría son profesores por asignatura), que fomenta la falta de compromiso con la universidad, la inquietud laboral y la vacilación en su vocación docente. En segundo lugar, la internacionalización debe implantarse decididamente en la contratación temporal y definitiva- de profesores, así como en la libre convocatoria de plazas docentes. En tercer lugar, debe potenciarse la preparación disciplinar y metodológica de los docentes, multiplicando programas de doctorado de calidad y exigiendo, a su vez, que ningún profesor acceda a ninguna plaza docente de nivel medio o alto si no ha completado antes su doctorado.

Hemos dedicado el último apartado del artículo al portal web de las universidades latinoamericanas. Como reflejan los resultados del "Ranking Web (Webometrics) de Universidades", elaborado por el Laboratorio de Cibermetría del CSIC, también en este terreno las universidades de América Latina cuentan con un amplio espacio de mejora. El portal web es la tarjeta de presentación de una institución universitaria, así como la principal plataforma de interacción con sus estudiantes -actuales y potencial-, con sus profesores y, lo que es también muy importante, con los diversos agentes del mundo empresarial, científico y académico que, a nivel nacional e internacional, están llamados a establecer relaciones creativas y beneficiosas con la universidad.

\section{Referencias}

Altbach, P. G., Reisberg, L. \& Rumbley, L. E. (2009). Trends in Global Higher Education: Tracking an Academic Revolution. Paris: UNESCO.

ARWU. (2015). Academic Ranking of World Universities. Recuperado de http://www.shanghairanking.com/es/ARWU2015.html

Bain, K. (2005). Lo que hacen los mejores profesores universitarios. Valencia: Publicacions de la Universitat de Valencia.

Brunner, J. J. \& Ferrada, R. (Eds.). (2011). Educación Superior en Iberoamérica: Informe 2011. Santiago de Chile: RIL Editores.

Centro Superior de Investigaciones Científicas (CSIC). (2016a). Ranking Web de Universidades: Metodología. Recuperado de http://www.webometrics.info/es/metodologia

Centro Superior de Investigaciones Científicas (CSIC). (2016b). Ranking Web de Universidades: Latinoamérica. Recuperado de http://www.webometrics.info/es/Latin_America_es

Gazzola, A. L. \& Didriksson, A. (Eds.). (2008). Tendencias de la Educación Superior en América Latina y el Caribe. Caracas: IESALC-UNESCO.

Llano, A. (2002). La Universidad ante lo nuevo. Recuperado de http://www.unav.es/noticias/llano. html

Ministerio de Educación Superior, Ciencia y Tecnología (MESCyT). (2011). Informe general sobre estadisticas de Educación Superior 20102011. Santo Domingo: Innovation Technology, S. A.

Nerad, M. \& Evans, B. (2014). Globalization and its impacts on the quality of PhD education: Forces and forms in doctoral education worldwide. Rotterdam: Sense Publishers. 
Quacquarelli Symonds. (2016a). QS University Rankings: Latin America. Recuperado de http:// www.topuniversities.com/university-rankings/ latin-american-university-rankings/2016

Quacquarelli Symonds. (2016b). QS University Rankings: Latin America. Methodology. Recuperado de http://www.topuniversities.com/ latin-america-rankings/methodology

Rojas, G. (Ed.). (2012). Textos fundamentales sobre la universidad. Santiago de Chile: Graphics Chile S. A.

Secretaría de Estado de Educación Superior, Ciencia y Tecnología (SEESCyT). (2008). Plan Decenal de Educación Superior 2008-2018: una educación superior de calidad para el país que soñamos los dominicanos. Santo Domingo: SEESCyT.

Sistema de Información de Tendencias Educativas en América Latina (SITEAL). (2011). Cobertura relativa de la educación pública y privada en América Latina. Recuperado de http://flacso.redelivre.org. br/files/2012/07/704.pdf

Steiner, G. (2007). Lecciones de los maestros. México D.F.: Siruela.

The Times Higher Education. (2016). The Times Higher Education World University Rankings 20152016. Recuperado de https://www.timeshighereducation.com/world-university-rankings

U.S. News and World Report. (2016). Best Global Universities Rankings. Recuperado de http://www. usnews.com/education/best-global-universities/ rankings

UNESCO Institute for Statistics. (2013). Global Flow of Tertiary-Level Students. Recuperado de http://www.uis.unesco.org/Education/Pages/international-student-flow-viz.aspx

UNESCO. (2015). América Latina y el Caribe. Revisión Regional 2015 de la Educación para Todos.
Recuperado de http://unesdoc.unesco.org/images/0023/002327/232701s.pdf

URAP. (2015). University Ranking by Academic Performance. Recuperado de http://www.urapcenter.org/2015/world.php?q=MS0yNTA=

\section{Datos de filiación}

Enrique Sánchez Costa (Barcelona, 1985) es licenciado en Humanidades por la Universidad Pompeu Fabra (UPF); magíster en Literatura Comparada: estudios sociales y culturales por la Universidad Autónoma de Barcelona (UAB) y doctor en Humanidades por la Universidad Pompeu Fabra (donde recibió el Premio Extraordinario de Doctorado). Ha realizado estancias de investigación en la British Library (Londres), la Library of Congress (Washington D.C.), la Bibliothèque Nationale de France (París) y la Biblioteca Nacional de Catalunya (Barcelona). Es autor de $E l$ resurgimiento católico en la literatura europea moderna (1890-1945) (Ediciones Encuentro: Madrid, 2014), cuya traducción inglesa publicará Peter Lang. Es profesor del Departamento de Español de la Pontificia Universidad Católica Madre y Maestra (PUCMM, Santo Domingo), donde ha impulsado el primer doctorado propio de la República Dominicana, el Doctorado en Estudios del Español: Lingüística y Literatura, del que es coordinador. 